\title{
FNN based Adaptive Route Selection Support System
}

\author{
Saoreen Rahman \\ Institute of Information Technology \\ Jahangirnagar University \\ Dhaka-1342, Bangladesh
}

\author{
M. Shamim Kaiser \\ Anglia Ruskin IT Research Institute \\ Anglia Ruskin University \\ Chelmsford, UK
}

\author{
Mahtab Uddin Ahmmed \\ Dept. of Mathematics \\ Jahangirnagar University \\ Dhaka-1342, Bangladesh
}

\begin{abstract}
This paper presents Fuzzy Neural Network (FNN) based Adaptive Route Selection Support System (ARSSS) for assisting drivers of vehicles. The aim of the proposed ARSSS system is to select path based on shortest possible time. The proposed system intakes traffic information, such as volume to capacity ratio, traffic flow, vehicle queue length and green cycle length, passenger car unit etc using different types of sensor nodes, remote servers, CCTVs and the road information such as path length, signalized junctions, intersection points between source-destination pair are captured using GPS service. A FNN has been employed to select an optimal path having shortest time. The input parameters of FNN are distance, signal point delay, road type and traffic flow whereas the output parameter is path selection probability which paves the way to identify the best suitable path. The simulation result revels that FNN based ARSSS outperforms more accurate than that of other route selection support system (webster delay model) and artificial neural network (ANN) in estimating path delay.
\end{abstract}

Keywords-GPS; Fuzzy Neural Network; Path delay; Signal Point Delay; Webster Delay Formula

\section{INTRODUCTION}

With the increase of metropolitan cities, traffic scenario has become more complex and heavily congested. The road traffic of metropolitan city includes different vehicle types, lane or non-lane base communication, signal points, cross-sections and so on, which introduces long traffic delay. It is a universal truth that people always want to reach their destination in shortest possible time. This human nature introduces the need of an Adaptive Route Selection (ARS) technique, which allows to discover the optimal route between a source-destination pair. An Adaptive Route Selection Support System (ARSSS) assists vehicles to find out the shortest distance path as well as shortest time path for a source-destination pair.

The estimation of traffic delay is one of major requirements for the establishment of ARS technique. There are two major types of traffic delay known as congestion delay and propagation delay. Propagation delay is a unit delay which depends on the path length and its corresponding speed. On the other hand, congestion delay is a random delay depends on various factors such as signalized junction, passenger car unit (PCU), queuing length, cycle length etc. Estimating these types of delay becomes more complicated for metropolitan cities as they include heterogeneous traffic environment [1][2].

Considering heterogeneous traffic environment, researchers have proposed variety of approaches. However, Most of the available delay estimation technique is used for homogenous traffic environment. But for heterogeneous traffic environment, more feasible, dynamic and real-time approaches should be considered. Keeping in mind of heterogeneous traffic environment, the introduction of artificial intelligence in this research field is more appreciated [3]. Fuzzy Neural Network (FNN) is suitable for uncertain or approximate reasoning which is capable of estimating solutions under certain information. FNN approach also makes a system more adaptive and reliable. As metropolitan cities include heterogeneous traffic environment, FNN is more applicable for traffic delay estimation and optimal route selection between a source-destination pair due to it's nature of adaptivity and flexibility. FNN based ARSSS has the capability of training and learning itself using expert knowledge system and making dynamic result for a sourcedestination pair.

A well known metropolitan city in Asian subcontinental region is Dhaka, capital of Bangladesh. Unlike other metropolitan cities, Dhaka also includes heterogeneous traffic environment. It needs to maintain non-lane based traffic environment with variety of traffic vehicles. It also supports different types of transportation with low traffic capacity and unstructured road map [4]. Under such circumstances of the city Dhaka, FNN based route selection support system can make its people capable of traveling between a sourcedestination pair in shortest possible time.

The motivation of the proposed work is to represent a concurrent ARSSS system which is well suited for heterogeneous traffic environment for Dhaka [23]. It intakes route map and traffic information dynamically, delivers optimal possible routes to its user between a source-destination pair. However to identify optimal route with minimum estimated time delay, all kinds of delay needs to be considered for all available routes between a source-destination pair. In a nutshell, it can be said that, the identification of optimal path considers not only distance factor but also time factor which can aid to a more adaptive system of route selection.

In this paper, a FNN based ARSSS is introduced. The proposed system consists of a centralized server, some remote servers which contain DBs, numerous sensors, CCTVs which collect road traffic information and GPS receivers to get latitude longitude information of source-destination points. When a driver submits route selection submits their request to the corresponding server and wait for the result. Here, GPS service is used for determining route information such as coordinates of source-destination points, path length, cross sections, signalized junctions, intersection points etc [5]. Path delay estimation 
is a major portion for optimal path identification. To calculate them a classic statistical model is used. To determine a route as an optimal solution FNN is used. It takes various information as input parameters, process them, estimates optimal route and generates the optimal path as output result. In this regard, to train FNN, hybrid learning algorithm is used which makes the performance of FNN more acceptable and realistic.

Based on the above conditions and by considering real time traffic data, the proposed system delivers the best available path which requires least travel time for a specified sourcedestination pair for its user.

The rest of the paper is organized as follows. Related works have been reviewed in Section II. System model is presented in Section III. System architecture is included in Section IV. Simulation and results are discussed in Section V. Finally our work is concluded in Section VI.

\section{RELATED WORKS}

In modern road traffic system the most focused problem is efficient and fastest path selection on a busy road. Time that is needed to travel between a source-destination pair, depends on various factors that affects the travel time. Such kinds of affect can be considered as car speed, intersection delay, signalized junction, lane capacity, heterogeneous traffic environment, path distance, queuing length etc. Moreover these factors kill valuable time of passengers [6]. For the above circumstances people have to choose the appropriate path directions from source to destination so that they can reach their destination in time. Efficient route selection system generally considers minimum delay time within source and destination. In a highly populated area one of the most important points of roads and streets are signalized junction. To calculate signalized junction some common measurements are considered like average delay per PCU, cycle length, PCU, green signal ratio, degree of saturation etc. within a path from source to destination [7].

By using deterministic queuing analysis, F. V. Webster, developed a series of traffic theories. His proposal included a statistical regression based model for delay estimation that is experienced by researchers at under saturated signalized intersections. Generally transportation professionals use vehicle delay as a parameter to observe the performance of signalized intersection. Due to random arrival of vehicles (Car, Bus, Mini Bus, Bus, Two-Wheelers) delay estimation is more complex, several populated models have been proposed to handle this complex issue [8] [9].

Among various traffic models, the most well-known model was developed by Miller and Akcelik in Australia [10], which used in HCM model in United States [11] and the model developed by Teply in Canada [12]. These models are logically superior to Webster's classical model because they can successfully deal with over saturated conditions and homogeneous traffic behaviours.

G.K.H. Pang, K. Takabashi, T. Yokota, proposed an approach named as adaptive route selection for dynamic route guidance system based on fuzzy-neural approaches [37]. In 2005, "Heuristic shortest path algorithms for transportation applications" has been proposed which was a survey review of various heuristic shortest path algorithms for traffic network
[13]. In 2007, Lefebvre and Balmer proposed an approach that computes a time-dependent shortest path for traffic network [14]. In 2010 and 2014, two different shortest path algorithms "Floyed algorithm" and "Dijkstra algorithm" were proposed [15] [16]. They optimized these algorithms to identify shortest path within specific nodes for various traffic networks. Gutenschwager, Axel Radtke, Volker and Zeller compared different approaches for traffic networks. They also implemented a model for automatic routing of vehicles [17]. In 2013 ANN based Short term traffic flow prediction for a non urban highway was proposed. In these paper author used past traffic data for short term traffic flow prediction [38]. In 2014 a work has been proposed that introduced highway dimensions and efficient shortest path algorithms [18]. For transportation in 2015 a route planning approach has been proposed which considered various techniques for identifying travel time delay within a particular source and destination [19].

However, most of these models are primarily focused on highways and systematic traffic systems that do not generally account with mixed-speed vehicles and multi-lane systems. These models are not suitable enough for a crowded metropolitan city like Dhaka, the capital of Bangladesh, where travelers experience difficulties in multi-lane roads due to chaotic nature of mixed-speed, as well as mixed-type, traffics and not so systematic traffic-controlling mechanisms. To address this problem, Hoque and Imran modified the Webster delay model to make it usable under non-lane based mixed road traffic condition [10]. The model has been calibrated to form a "Modified Webster Delay Formula", which was subsequently validated by comparing the expected delays with observed delays.

In 2009, Chu Cong, Tran Hoai, Tran Thanh and Kazushi proposed an expectation function and Taylor series for Webster formula that estimate delay at pre-timed signalized intersections under heterogeneous traffic conditions [21]. In 2014, Hadiuzzaman, Mizanur, Tanweer and Ahsanul developed heterogeneous traffic model at signalized intersection. Based on field measurement they provide a regression analysis which measures the accuracy of traffic models considering the traffic scenario of Dhaka City [22]. The work of [23] presents a traffic information based adaptive route selection support system by offering a model that helps to calculate the minimum delay for a particular source to destination according to the google map.

Some traffic model designers consider FNN approach as an adaptive solution for route selection. Now-a-days, for the establishment of an ARSSS, FNN have considered one of the most eligible technique for implementing an intelligent route selection support system [1] [26]. In 2011, a bioinspired integrated with FNN introduced a dynamic route selection system [27]. Following that year, a PSO based dynamic shortest path algorithm using fluid neural network was proposed. That approaches were especially applicable for stochastic traffic network [28]. After few years later, a hybrid optimization algorithm has been proposed which introduced ASO and FNN for better result [29]. A work of ANN based road traffic accidents prediction was introduced in 2014. This work is accomplished based on Jordan [36]. In 2014, R. Yasdi proposed an approach that predicts road traffic using neural network [39]. In 2015, a passenger route selection model 


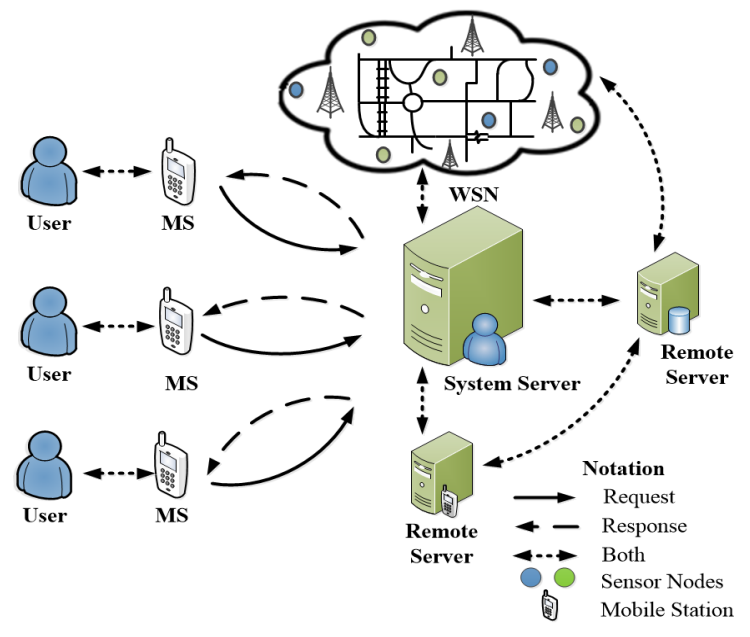

Fig. 1. System Model Scenario.

has been proposed by Fatma Al-Widyan, Nathan Kirchner, Michelle Zeibots [40]. From 2010 to 2016, several FNN based approaches have introduced in work [33][34][35].

In this paper, we use the proposed model scheme of [23] for more realistic modeling of FNN based route selection support system for Dhaka, Bangladesh.

\section{SySTEM MODEL}

This section describes the system model, work flow and formulation of the ARSSS.

Figure 1, shows the system model of ARSSS. The entire system can be considered as a real-time client server approach. ARSSS includes users, system server, several remote servers and Wireless Sensor Network (WSN). It uses variety of information which are collected from GPS, CCTVs and others existing real-time remote servers. ARSSS also captures data from sensor nodes which are supported by WSN. The system server takes service request from it's client via smart device (mobile phone), generates the result of the service and responses back to its requesting client. In another word, for a source-destination pair, the proposed ARSSS takes predefined features as inputs from it's user, processes them using captured information and delivers the best available path as output. The main aim of the proposed system is to define an optimal route among several possible routes between a source-destination pair. Thus, the scenario shown in figure 1 , is considered to provide the shortest distance path as well as shortest time path which is optimal path between a source-destination pair.

\section{A. System Work flow}

In this paper, a heterogeneous traffic environment is considered which includes mixed-speed, mixed-type vehicles and multi-lane road structure. The work flow of the proposed system is shown in Figure 2.

At the very beginning, the proposed system considers user preference, denoted by $\Phi$, and source-destination pair, denoted by $(s ; d)$, as inputs which is defined by users. $\Phi$ offers users to declare the presence of priority for a particular route between a source-destination point. Whereas, the other input, $(s ; d)$ offers users to declare the source-destination names or locations. That is, $(s ; d)$ takes GPS coordinates (latitudes, longitudes) of user defined source-destination pair. Moreover, each user provides position and movement information to the corresponding server in background mode using GPS. After having basic information (latitudes, longitudes, user-preference) captured form user, it runs query to other information holder nodes (servers, other users, sensor nodes) for capturing other information to generate optimal route between two predefined nodes. A volatile storage is maintained to store concurrent data for the upcoming calculation. After capturing input parameters, a pre-decision phase is considered. Next, based on the decision of previous phase, data per-processing phase is performed. Regarding source destination points it calculates all possible delays for all possible routes using GPS map. After completion of data pre-processing phase, FNN controller identifies an optimal route between a source-destination pair. After that, path identification phase is performed. This phase considers the resulted route of previous phase as the optimal route and declares it as the best one. Finally, the proposed system delivers the identified optimal route to its user and stores the solution for further usage.

\section{B. Initial Decision Method}

Initially, the proposed system takes user preference $\Phi$ and source-destination points $(s ; d)$ these two major parameters as input from the user. To identify an optimal path for a particular location, user must have to define specific source-destination point. Using GPS coordinate service the latitudes and longitudes of user defined source and destination points are determined [30] [31][32]. Therefore, $(s ; d)$ input parameter can be functioned as:

$$
\begin{aligned}
& s=\left(\text { lat }_{s}, \operatorname{long}_{s}\right) \\
& d=\left(\text { lat }_{d}, \operatorname{long}_{d}\right)
\end{aligned}
$$

Here, $s$ and $d$ are considered as source-destination locations whereas lat and long are considered as latitude and longitude coordinate values of a source-destination point.

Another major consideration as input parameter is user preference, denoted by $\Phi$ which can be declared as

$$
\Phi \quad \epsilon \quad[0,1]
$$

Here, $\Phi=1$ means user declares high level of user preference in choosing route between source-destination pair; $\Phi=0.5$ means user has confusion in choosing route between source-destination pair and $\Phi=0.0$ means user has no preference in choosing route between source-destination pair.

If $\Phi$ results in high level of user preference then the selected route chosen by the user will be considered as the best path. In this case, no further calculation and decision making is required. For the other two cases the proposed system will calculate total delay time for each possible route and propose a reliable decision by identifying the optimal path between a particular source-destination point. To generate and identify the optimal route several phases have been considered which have been discussed in the upcoming sections. 


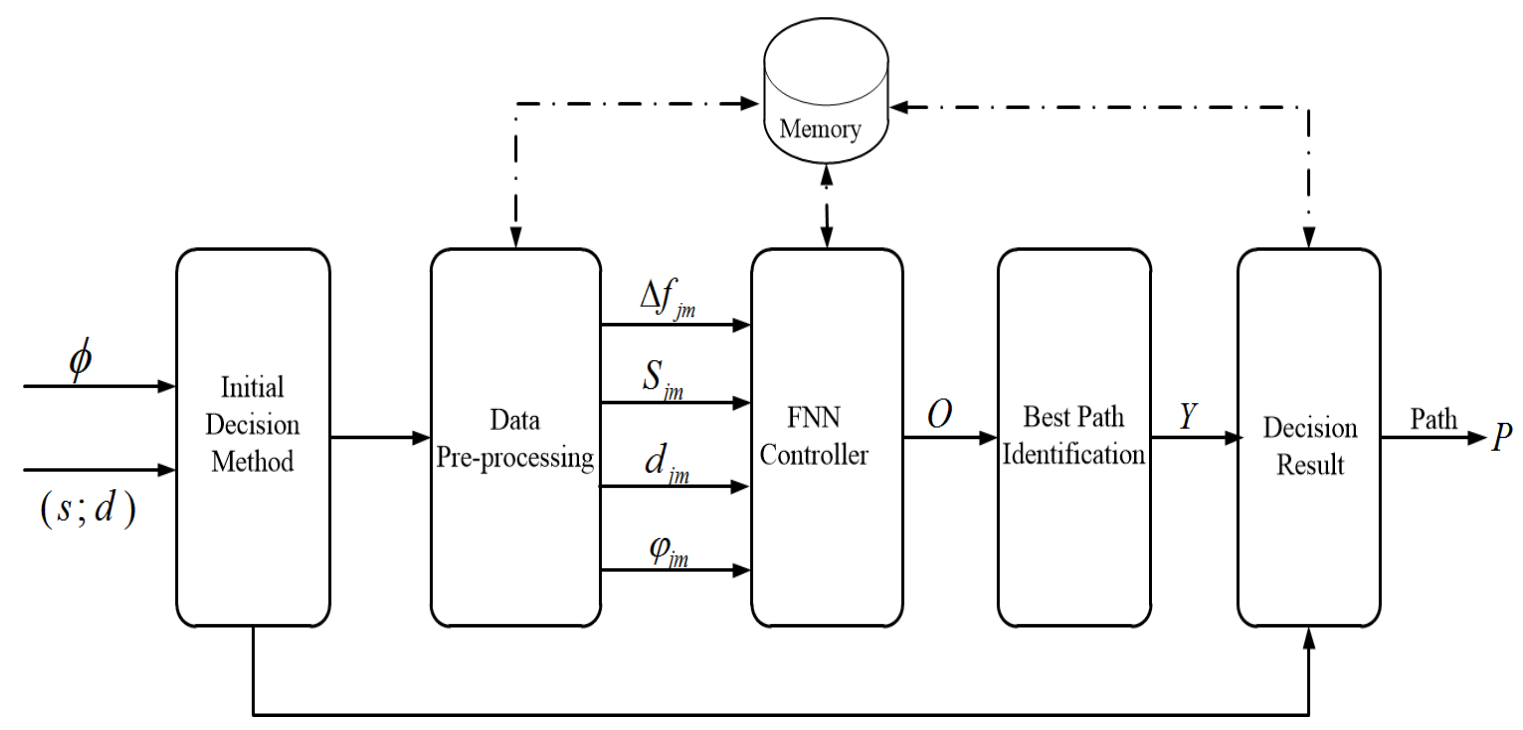

Fig. 2. System Work flow.

\section{Data Pre-processing}

These phase performs two major activities in optimal path selection process. First one is capturing all necessary information that are required to determine optimal path for a source-destination pair. It collects concurrent usable information from the user in background mode using GPS[30] [31]. It also runs query to other nodes to get required information for further calculation for optimal route selection. All information captured by this phase are memorize and updated in a volatile storage.

And second one is performing delay calculation for all possible routes between a source-destination pair using GPS map. To calculate the total delay time from a particular sourcedestination pair straight path delay, cross section delay and signal point delay is considered. The straight path delay is the time that needed to cover a specific distance. The cross section delay is the time that a vehicle takes to cross a crossingpoint intersection. The signal point delay is the time which is required at a signalized junction [23] [24] [25]. It also considers road type and traffic flow calculation used in FNN controller.

1) Path Delay: Path delay includes both straight path delay as well as cross section delay. It considers path distance along with referred path speed both for straight path and a crosssection point. If $j$-th route with $m$ branches is chosen to cover the distance $d_{j m}$ with average speed $v_{j m}$, then the path delay can be denoted by $D_{j m}$ and defined as:

$$
D_{j m}=\sum_{m=1}^{m} d_{j m} / v_{j m}
$$

Where, $j, m \varepsilon \Re: \Re \leftarrow$ real_numbers.

2) Signal point Delay: Signal point delay refers the average delay per vehicle at a signalized junction of a particular route with several existing branches. In this paper, signal point delay calculation is considered in two steps. First one is the calculation of delay time for a particular signalized intersection followed by modified Webster Delay Model. Another one is variance calculation for delay time in a particular signalized intersection followed by Taylor Series. The estimated time for signal point delay for $j$-th route with $m$ branches, can be expressed as:

$$
t_{j m}=\xi+\Delta_{T}
$$

$t_{j m}$ calculates congestion delay time at a signalized junction. It includes two basic terms such as $\xi=$ Average delay time. $\Delta_{T}=$ Variance of delay time.

In Equation (17), First term $\xi$ denotes average delay calculation for a signalized junction and second term $\Delta_{T}$ denotes variance of signal point delay for that particular signalized junction.

Average delay time is considered in the first term. This paper uses the modified Webster delay model which is discussed in [10]. Therefore, the average delay time at any signalized junction is denoted by $\xi$. It can be calculated by the following equation.

$$
\xi=\frac{c(1-\lambda)^{2}}{2(1-\lambda x)}+\frac{x^{2}}{2 v(1-x)}+\rho
$$

Where,

$c=$ Cycle length of green signal. $v=$ Vehicle flow rate (Passenger Car Unit/hour). $\lambda=$ Effective green ratio. $x=$ Degree of saturation (volume to capacity ratio).

In Equation (6), the first term represents the average delay to the vehicles assuming uniform arrivals. The second term represents the additional delay due to the random arrivals. The third term is a semi-empirical adjustment term used to maintain accuracy for specific field conditions of vehicle arrivals. The modified Webster delay formula is used to estimate signalized intersections for non-lane based heterogeneous traffic conditions.

The expression for delay, given by Equation (6) was not 
derived entirely theoretically. The first and second terms have a theoretical meaning, but the last one is purely empirical. The modification of Websters delay model under non-lane based heterogeneous traffic condition can be measured by adding an empirical adjustment term with first two theoretical terms.

Paper [10] introduced an adjustment term specified for heterogeneous traffic conditions like as dhaka city. In Equation (6) the adjustment term is denoted by $\rho$. According to the modified Webster delay model, the adjustment term, $\rho$, can be expressed by,

$$
\rho=\alpha_{0}+\alpha_{1} q+\alpha_{2} c+\alpha_{3} x+\alpha_{4} \lambda+\alpha_{5} \eta
$$

Where, $\alpha_{0}=$ Constant term in the model. $\alpha_{j}=$ the weighted coefficient for corresponding term.

The equation (7) adjusts the validity of the Webster delay model for Dhaka city with some measurement of field observations. This correction term is taken for best suited local road situation and it dependents on all the previous variables which is unique for non-lane based road traffic and can be measured from field observation data.

Variance of delay time is measured in the second term . The term variance is considered which is followed by Taylor Series. For non linear function $Y=f(X)$, the Taylor Series(1979) is,

$$
Y=f\left(\mu_{x}\right)+\left(X-\mu_{x}\right) \frac{d^{2} f}{d X^{2}}+\frac{1}{2}\left(X-\mu_{x}\right)^{2} \frac{d^{2} f}{d X^{2}}
$$

Where, $\mu_{x}=$ Mean value. $X=$ Random variable.

The expected value can be derived by the the second order of Taylor Series.

$$
E(Y)=\frac{1}{2} \operatorname{Var}(X) \frac{d^{2} f}{d X^{2}}
$$

Where, $\operatorname{Var}(X)=$ the variance of $X$ and estimated by,

$$
\operatorname{Var}(X)=E\left(X^{2}\right)-\mu_{x}
$$

Here, $E\left(X^{2}\right)=$ the expectation value. $\mu_{x}=$ Mean of $x$.

The variance of $Y=f(X)$ can be expressed as:

$$
\begin{aligned}
\operatorname{Var}(Y) & =\operatorname{Var}(X)\left(\frac{d f}{d X}\right)^{2}-\frac{1}{4} \operatorname{Var}(X)^{2}\left(\frac{d^{2} f}{d X^{2}}\right)^{2} \\
& +E\left(\left(X^{2}\right)-\mu_{x}\right)^{3} \frac{d f}{d X} \frac{d^{2} f}{d X^{2}} \\
& +\frac{1}{4} E\left(\left(X^{2}\right)-\mu_{x}\right)^{4}\left(\frac{d^{2} f}{d X^{2}}\right)^{2}
\end{aligned}
$$

The expectation function method is an analytic procedure to overcome shortcomings of estimation procedure. It uses estimate functions which depends on random variable $X$. In this study, for the modified Webster delay model, the degree of saturation $x$ is considered as random variable.

Considering Taylor Series, $\Delta_{T}$ calculates the variance of delay time for modified Webster Delay model. It indicates how far measured delay time can spread out for particular signalized intersection point. In another word, variance calculates the saturation for estimated value and measured delay time value.
Thereby, $\Delta_{T}$, can be expressed as,

$$
\Delta_{T}=1 / 2 p_{x_{j}} q_{x_{j}}
$$

Here,

$$
p_{x j}=\frac{c(1-\lambda)^{2} \lambda^{2}}{(1-\lambda x)^{3}}
$$

and

$$
q_{x j}=\mu_{x}^{2}\left(1+C V^{2}\right)-\mu_{x}
$$

Where, $C V=$ Covariance of correlation. $\mu_{x}=$ mean of degree of saturation, $x$.

Equation (6) is used to calculate delay time for a signalized junction. And Equation (12) is used to calculate the variance of estimated delay time for that particular signalized junction. Thus, Equation (17) calculates signal point delay for a particular signal point intersection.

Therefor, the total delay time for $m$ branches at different signal point can be expressed as:

$$
S_{j m}=\sum_{m=1}^{m} t_{j m}
$$

Total Delay Calculation: Total delay for an individual path with $m$ branches for a source-destination pair can be expressed as:

$$
\tau_{j m}=D_{j m}+S_{j m}
$$

Where, $D_{j m}=$ path delay for branch $m$ of $j$-th route. $S_{j m}$ = signal point delay for branch $m$ of $j$-th route.

By using Equation [15] the total delay time of a particular path for a source-destination pair can be measured. It consists of two distinct terms. By using Equation [3], first one calculates all path delay for a particular path. And the last one calculates signal point delay of all signalized junctions for that specific route/path followed by Equation [14].

3) Road Type: Road type parameter considers route category as an express way or high way. As express way requires travel payment, less vehicles prefer to use that route. However, some public transports and private vehicles wish to travel on that route. On the other hand, highway does not require any travel payment which is usually preferable by all types of vehicles. Thus, it can be considered that express way has more traffic flow that that of high way. That is, high way includes more vehicles than that of express way. Therefor, road type parameter for route $j$-th with branch $m$ denoted as $j m$ can be defined as $\varphi_{j m}$, which is considered as:

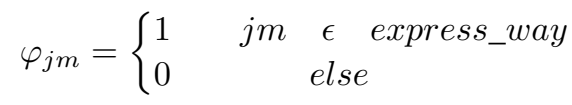

4) Traffic Flow: Traffic flow parameter is regarded as traffic load for a particular path with several branches. To identify the optimal path, selection of branches with shortest delay time is equivalently important. Traffic flow defined as $\Delta f_{j m}$ considers difference between inward vehicle flow, $\zeta_{j m_{i n}}$ as well as outward vehicle flow, $\zeta_{j m_{\text {out }}}$ of a particular route. Thus, the higher the traffic flow, the high the possibility of traffic load and the lower the traffic flow, the lower the possibility of 


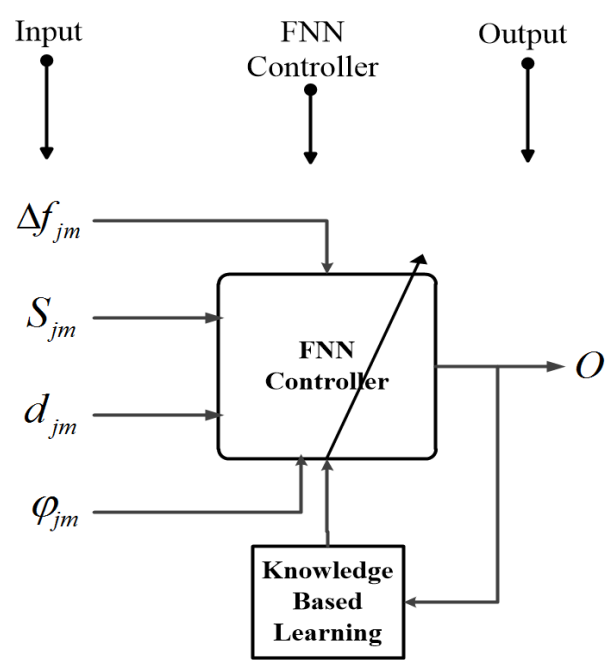

Fig. 3. Block Diagram of FNN_Controller Module.

traffic load. Therefore, the system selects that branch which includes least most traffic flow as well as traffic load. Traffic flow, $\Delta f_{j m}$ for route $j$ with branch $m$, can be considered as below:

$$
\Delta f_{j m}=\zeta_{j m_{\text {in }}}-\zeta_{j m_{\text {out }}}
$$

\section{FNN Controller}

In this section we discuss the phase named FNN controller.

1) Block Diagram of FNN Controller Module: These controller uses FNN approach for ARSSS. It includes fuzzy neural network for decision making to identify optimal route. FNN controller takes distance denoted as $D_{j m}$, signal point delay denoted as, $S_{i j}$, traffic flow denoted as $\Delta f_{j m}$ and road type $\varphi_{j m}$ as input parameters and performs fuzification-defuzification calculation and generates the best suitable route between a source-destination pair. Block diagram of FNN controller module is shown in figure 3.

\section{E. General Structure of FNN Controller}

Figure 4 shows the general structure of FNN controller for ARSSS. The Fuzzy Neural Network, i.e., FNN model, maps the inputs using input membership function (preceding parameters) into the output using output membership function (consequent parameters). Based on the input features the FNN controller generates the probability of route selection for a source-destination pair. Both the preceding and consequent parameters are adjusted via the learning process. The hybrid of back propagation and least square estimation are used. Based on the chosen error criterion (which is the sum of squared difference between actual and desired outputs), the FNN changes the input and output membership function parameters. Considering our case, FNN is a five-layers fuzzy neural network that simulates the working principle of a fuzzy inference system . The linguistic nodes in layer one and four represent the inputs and output linguistic variables, respectively. There are four input parameters which are $X_{1}$ as $D_{j m}$ (distance), $X_{2}$ as $S_{i j}$ (signal point delay), $X_{3}$ as $\Delta f_{j m}$ (traffic flow) and $X_{4}$ as $\varphi_{j m}$ (road type). These four input parameters results

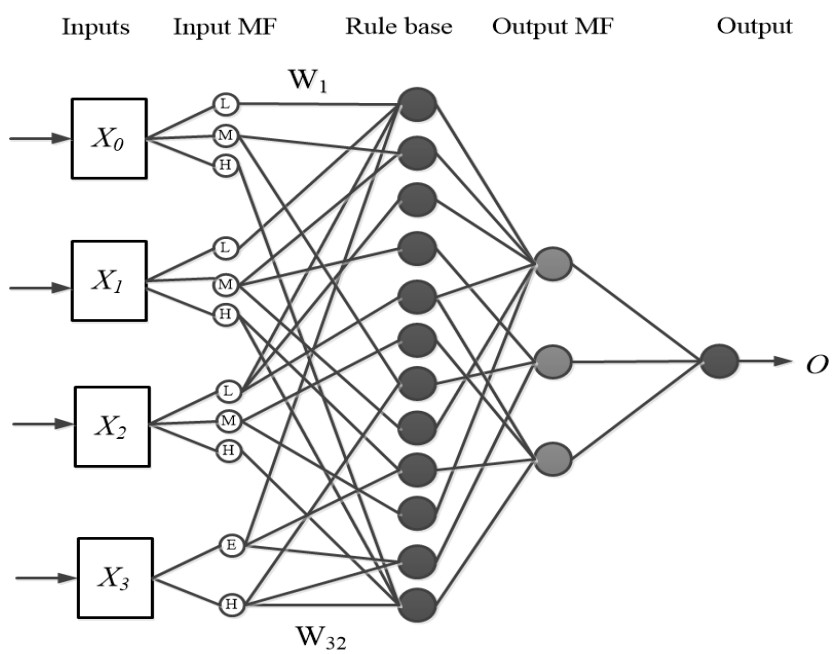

Fig. 4. General Structure of FNN Controller.
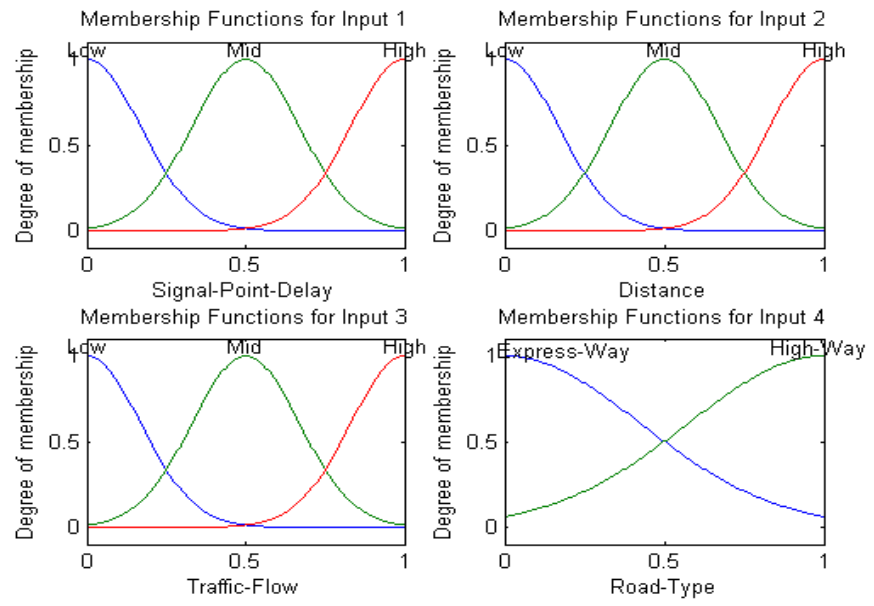

Fig. 5. Membership functions for all input parameters.

in a single output which is path selection probability denoted as $O$. Nodes in layers two acts as membership functions for input variables and the membership function used in FNN is generalized gaussian membership function. It can be noted that, first three input parameters includes three membership functions whereas the last one includes only two membership functions. Each neuron in the third layer represents one fuzzy rule. FNN used in ARSSS includes 32 fuzzy rules. Here, The third Layer normalizes the strength of all the rules whereas the forth layer used gaussian membership membership function to aggregated results which are forward to the output layer as path selection probability, $O$.

1) Membership Function for all Inputs: Figure 8 shows four individual plots of membership functions for all input parameters. Figure 8-(a)(b)(c) include fuzzy set [Low, Medium, High] which is applicable for $D_{j m}$ (distance), $S_{i j}$ (signal point delay), $\Delta f_{j m}$ (traffic flow) these three input parameters. Whereas, Figure 8-(d) includes fuzzy set [Express way, High way] which is only applicable for input variable $\varphi_{j m}$ (road type) from input layer. All the membership functions were derived from the fuzzy set of input parameters where gaussian 


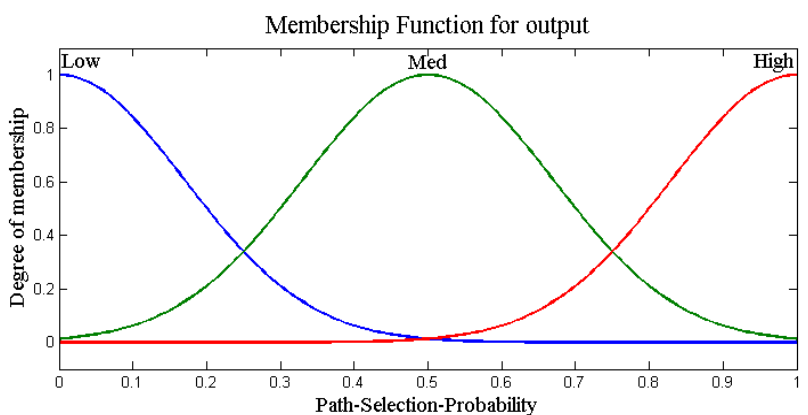

Fig. 6. Output membership function.
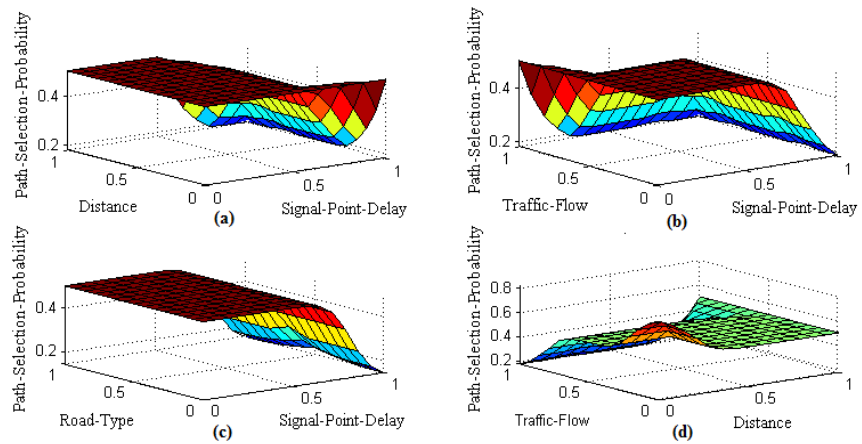

Fig. 7. Surface view of rule base system.

membership function is adopted for transforming the crisp input into linguistic level.

2) Membership Function for Output: Figure 9 shows membership function for output parameter path-selectionprobability, $O$. It incudes fuzzy set [Low, Medium, High]. The output membership function is derived by output fuzzy set. When a route holds all features of a best path then the probability of that path selection is high. Otherwise it is low or medium based on the features that it holds. Here, gaussian membership function is considered for output membership function.

3) Surface View: Figure 10 shows four individual plots of surface view of input-output parameters for the proposed system. Here, Both $x$-axis and $y$-axis represent input parameters whereas $z$-axis represents the output parameter. Figure 8-(a) shows the surface view of input parameters signal point delay, distance with output parameter path selection probability, $O$. Followed by figure 8-(a), 8-(b)(c)(d) show the surface view of input parameters between traffic flow, signal point delay; road type, signal point delay and traffic flow, distance with output parameter path selection probability.

\section{F. Best Path Identification and Decision Result}

This phase takes the output of FNN controller. After completing activities of FNN controller, these phase captures the output $O$ (path selection probability) which declares the probability of each available routes between a source-destination pair. It takes that probability result, identifies an optimal route denoted by $Y$, which includes shortest path delay as well as shortest time delay. At the end of this phase, best suited path $P$ is defined and send back to it's user as a solution of system query. Once a solution is declared for a specific sourcedestination pair, the resulted optimal path is stored in memory and being updated or modified while it is needed.

\section{System ARChitecture}

In this section we present the system architecture of our proposed system. First we present an algorithm that supports our system for generating the best possible result. Then we discuss the block diagram of FNN controller which performs decision making of path selection for the proposed adaptive scheme.

\section{A. Algorithm}

In this section we propose an algorithm that declares the procedure, step by step, to identify best path between a source-destination point. The algorithm used in our proposed scheme is presented in Algorithm 1

\section{Simulation And Results}

In this section we discuss the simulation environment used for our proposed adaptive system, and also the results estimated by the scheme. Here, the simulation model is created using MATLAB. For a user-defined source-destination pair, necessary data is fed to model and result is generated to get the most optimum choice of route.

\section{A. Simulation Parameters}

The simulation parameters used in ARSSS is given in Table I. For simplicity, we assume that the source-destination pair is fixed, e.g. the source-destination pair in Figure 1 for Panthapath $(23.7515 ; 90.3861)$ and Shahbagh $(23.7381 ; 90.3953$ in Figure 2 respectively. For a route, our proposed scheme takes all necessary parameters as inputs from the database. The values of different input parameters are varied within a range to analyze the effect of different road and traffic condition. From Table I, $d_{j m}$ and $v_{j m}$ are used for calculating path delay and the remaining parameters are used for signal point delay calculation. Any values that are not shown in the Table I are directly acquired from [10].

\section{B. Simulation Analysis in Route Selection}

In this section we discuss the simulation environment for optimal route selection. For the required timing estimation, we consider seven available paths as described in section IVA using the parameters from Table I. Several parameters are also varied to analyze the impact of different traffic load and road condition.

1) Effects of traffic load: Figure 8 shows the effect of traffic on the seven paths between source-destination pair as described in Section III. It has been found that the delays are different for different paths as the total distances are different. The time delay is proportional to distance if there is no traffic. But the delay changes based on the traffic saturation on the path as greater traffic hinders the movement and slows down average speed and thus increasing the required time. This calculation includes traffic delay for straight paths, cross section points and signal points. 


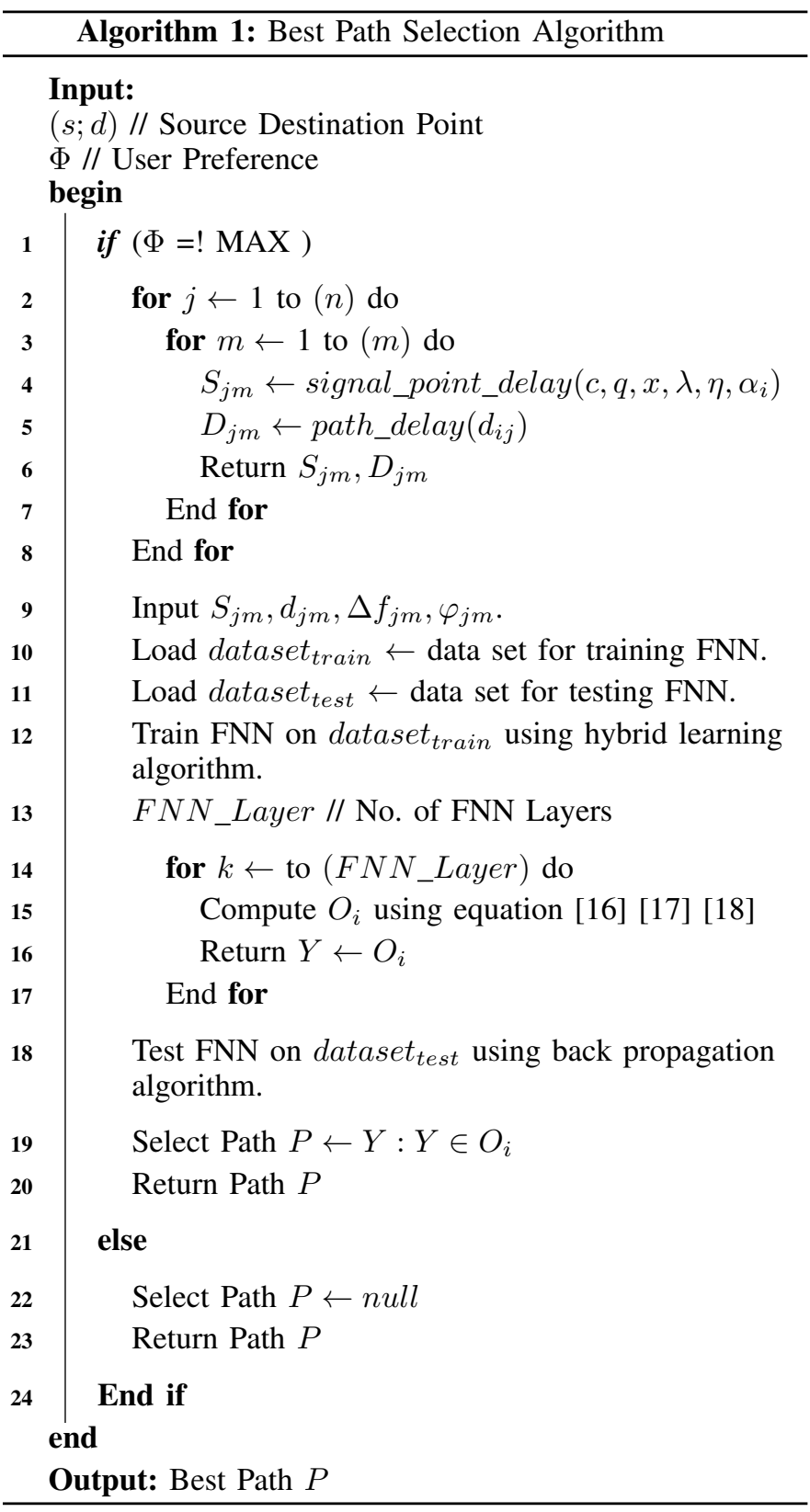

TABle I. Parameters of Straight Path Delay, Cross Section Delay and Signal Point Delay

\begin{tabular}{|c|c|}
\hline Parameter & Value \\
\hline$d_{j m}$ & {$[0.5-2.2] \mathrm{km}$} \\
\hline$d_{j m}$ & {$[40,50,60] \mathrm{kmh}^{-1}$} \\
\hline$c$ & $540 \mathrm{sec}$ \\
\hline$q$ & $1(\mathrm{PCU} / \mathrm{sec})$ \\
\hline$x$ & $0.20-0.28$ \\
\hline$\eta$ & 0.30 \\
\hline$\lambda$ & $<1$ \\
\hline$\alpha_{0}$ & $0.19-0.22$ \\
\hline$\alpha_{i}$ & $0.2-0.5$ \\
\hline$\Delta f_{j m}$ & $0.5-0.20$ \\
\hline
\end{tabular}

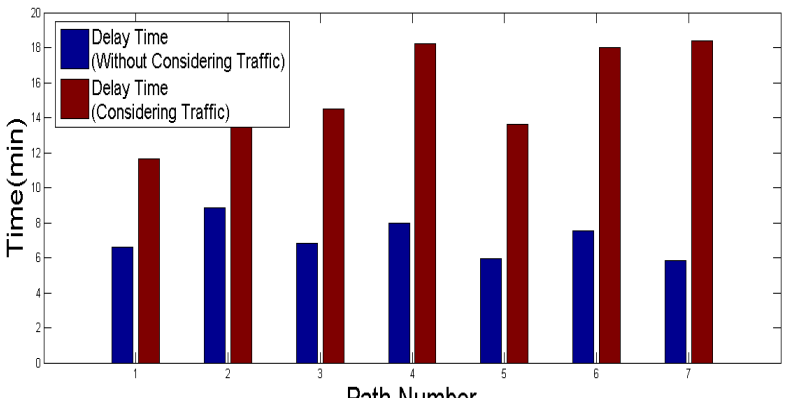

Path Number

Fig. 8. Effects of S-D paths distance with or without traffic on path delay

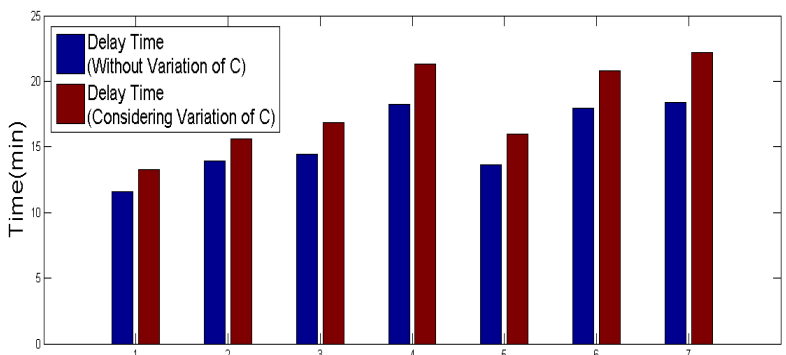

Path Number

Fig. 9. Effect of cycle length on path delay

TABLE II. Total Path Delay

\begin{tabular}{|c|c|c|c|}
\hline$j^{t h}$ Path & $D_{j m}$ (minute) & $S_{j m}$ (minute) & $\tau_{j m}$ (minute) \\
\hline $\mathbf{1}$ & $\mathbf{6 . 6 7}$ & $\mathbf{4 . 9 4}$ & $\mathbf{1 1 . 6 1}$ \\
\hline 2 & 9.08 & 4.84 & 13.93 \\
\hline 3 & 7.00 & 7.45 & 14.47 \\
\hline 4 & 8.15 & 10.06 & 18.23 \\
\hline 5 & 6.08 & 7.55 & 13.64 \\
\hline 6 & 7.74 & 10.33 & 17.97 \\
\hline 7 & 5.81 & 12.58 & 18.39 \\
\hline
\end{tabular}

2) Effects of Cycle Length: Figure 9 shows the effect of cycle length on the seven paths between source-destination pair. The illustration shows that cycle length has direct effect on the path delay. It is additive to total delay.

3) Effects of Degree of Saturation: Figure 10 shows the effect of degree of saturation on the seven paths between source-destination pair. It has been found that the degree of saturation has negligible effect on the total path delay, when considering large saturated traffic in the metropolitan area.

4) Route Selection: Table II shows the required time for traveling from source to destination via seven paths. Based on the given parameter, our proposed scheme chooses Pathl to be the best one in terms of minimum time required to travel. It should be noted that both straight path delay and signal point delay play the major role in final path delay whereas cross section delay may be neglected upto a certain magnitude. Based on this result further adjustment can be made in route selection as well as in the overall traffic system. 


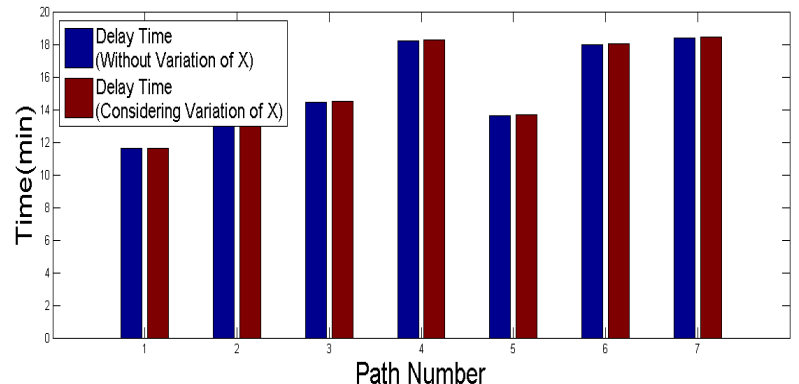

Fig. 10. Effect of degree of saturation on path delay

TABLE III. Performance ANAlysis of OUtPut Result

\begin{tabular}{|c|c|c|c|c|}
\hline Path & $\begin{array}{l}\text { Actual } \\
\text { Output }\end{array}$ & $\begin{array}{l}\text { Estimated } \\
\text { Output }\end{array}$ & $\begin{array}{l}\text { ANN Ap- } \\
\text { proach }\end{array}$ & $\begin{array}{l}\text { FNN } \\
\text { Ap- } \\
\text { proach }\end{array}$ \\
\hline 1 & 13.20 & 11.61 & 11.58 & 11.50 \\
\hline 2 & 16.00 & 13.93 & 13.80 & 13.60 \\
\hline 3 & 17.17 & 14.47 & 14.31 & 14.20 \\
\hline 4 & 23.53 & 18.23 & 18.10 & 17.90 \\
\hline 5 & 16.56 & 13.64 & 13.80 & 13.60 \\
\hline 6 & 19.58 & 17.97 & 17.80 & 17.60 \\
\hline 7 & 19.25 & 18.39 & 17.50 & 17.40 \\
\hline
\end{tabular}

TABLE IV. ERRors in OUtPut RESUlt ESTIMATION

\begin{tabular}{|c|c|c|}
\hline Path & ANN Approach & FNN Approach \\
\hline 1 & 0.14 & 0.01 \\
\hline 2 & 0.16 & 0.02 \\
\hline 3 & 0.2 & 0.019 \\
\hline 4 & 0.3 & 0.018 \\
\hline 5 & 0.2 & 0.00 \\
\hline 6 & 0.1 & 0.021 \\
\hline 7 & 0.1 & 0.057 \\
\hline
\end{tabular}

\section{Performance Analysis}

In this section we discuss performance analysis of our proposed system in. Table III shows the time delay of actual output without any intelligent system, estimated output by Webster delay model, ANN approach and FNN approach. It has been found that the proposed FNN outperforms the all the other mentioned methods. Table IV shows the error is the delay estimator. It has been found that the accuracy of the FNN approach is higher than that of the ANN approach.

\section{CONCLUSION}

This paper presents an ARSSS for a source-destination pair under heterogeneous traffic environment. The Dhaka city is considered to validate the proposed model. ARSSS includes different phases such as initial decision method, data preprocessing, FNN controller and best path identification for a particular source-destination pair. FNN controller takes sourcedestination pair distance, signal point delay, traffic flow and road type as input parameters and selects best path for a particular source-destination pair. These input parameters are captured by distributed wireless/wired sensor nodes, remote servers, CCTVs and above all GPS service. This work can be extended by including bio-inspired algorithm instead of FNN for Adaptive road selection support system.

\section{REFERENCES}

[1] Robert L. Bertini and Ahmed El-Geneidy, "Advanced Traffic Management System Data", Proceedings of 92nd Annual Meeting of Transportation Research Board, Washington DC, USA, January 13-17, 2013.

[2] Chaudhry, M. S. and Ranjitkar,"Capacity and Signal Timing Analysis of Signalized Intersections with Increasing Saturation Flow Rate", Proceedings of 92nd Annual Meeting of Transportation Research Board, Washington DC, USA, January 13-17, 2013.

[3] Iqbal, M.A., Zahin, A , Islam, Z.S. , Kaiser, M.S. "Neuro-Fuzzy based adaptive traffic flow control system", Communications, Devices and Intelligent Systems (CODIS), International Conference on, Kolkata, India, December 28-29, 2012. pp. 349 - 352, ISBN:978-1-4673-4699-3.

[4] Robert L. Bertini and Ahmed El-Geneidy. "Traffic Flow Interruptions in Dhaka City: Is Smooth Traffic Flow Possible?", 2013 Journal of PU, Part: B, Presidency University, India, July 13-17, 2013, Vol.2 , No.2, pp.46-54, ISSN: 2224-761.

[5] Akanbi A. K, Agunbiade O. Y., "Integration of a city GIS data with Google Map API and Google Earth API for a web based 3D Geospatial Application", International Journal of Science and Research (IJSR), Novembor 23-27, 2013, Vol.2 , No.11 ISSN (Online): 2319-7064.

[6] Chaudhry,M. S., Ranjitkars ,P., Wilson, D. J. and Hadas, Y., "Investigation of Queue Discharge Behavior at Signalized Intersection based on Analytical and Microsimulation Models", Proceedings of 90th Annual Meeting of Transportation Research Board, Washington DC, USA, January 23-27, 2011.

[7] Akcelik, R, "Traffic Signals:Capacity and Timing Analysis",Research Report ARR No.123, Australian Road Research Board, Victoria, Australia,Issue (July 2003), pp. 3-4.

[8] DingXin Cheng, Carroll J. Messer ,Zong Z. Tian, Juanyu Liu, "Modification of Webster Minimum Delay Cycle Length Equation Based on HCM 2000", Transportation Research Board for Presentation and Publication at the 2003 Annual Meeting in Washington, D.C., Texas Transportation Institute, Issue (July 2003), pp. 3-4.

[9] Webster, F. V., Traffic signal settings, Road Research Technical Paper No. 39, Road Research Laboratory, England, published by HMSO, 1958.

[10] N. M. Akcelik R, Rouphail, "Over flow queues and delays with random and platooned arrivals at signalized intersections", Journal of Advance Transportation, Vol.3, pp. 257-251, Issue (1994).

[11] TRB (Transportation Research Board), "Highway Capacity Manual", National Research Council, Washington D.C., USA, 2000.

[12] Teply, S., D. I. Allingham, D. B. Richardson and B. W. Stephenson, Canadian Capacity Guide for Signalized Intersections, Second Edition (S. Teply, ed.), Institute of Transportation Engineering, District 7, Canada, 1995.

[13] L. Fu, D.Sun, L.R. Rilett, "Heuristic shortest path algorithms for transportation applications: State of the art", Communications, Computers and Operations Research 33 (2006) 3324 334, May 3, 2005. pp. 1-3.

[14] Nicolas Lefebvre, Michael Balmer, "Fast shortest path computation in time-dependent traffic networks", Working Papers Traffic and Spatial Planning, June 2007, pp. 3-4, ISBN:978-1-4673-4699-3.

[15] Dachuan Wei, "An Optimized Floyd Algorithm for the Shortest Path Problem ", Journal of Networks, Vol. 5, No. 12, December 2010, pp. $1496-1497$.

[16] K. Rohila, P. Gouthami, Priya M, "Dijkstras Shortest Path Algorithm for Road Network", International Journal of Innovative Research in Computer and Communication Engineering, Vol. 2, No. 10, Octobor 2014, pp. 349 - 352, ISSN: 2320-9798.

[17] Kai Gutenschwager, Axel Radtke, Sven Volker, Georg Zeller, "The Shortest Path: Comparison of Different Approaches and Implementations for the Automatic Routing of Vehicles", 2012 Winter Simulation Conference, Octobor 2012, pp. 3312 - 3314, ISSN: 978-1-4673-4780-8. 
[18] Ittai Abraham, Daniel Delling, Amos Fiat, Andrew V. Goldberg, Renato F. Werneck, "Highway Dimension and Provably Efficient Shortest Path Algorithms", Microsoft Research, Microsoft Corporation, One Microsoft Way, Redmond, WA 98052, May 2014, pp. $2-3$.

[19] Hannah Bast, Daniel Delling, Andrew Goldberg, Matthias MullerHannemann, Thomas Pajor, Peter Sanders, Dorothea Wagner, Renato F. Werneck, "Route Planning in Transportation Network", International Journal of Innovative Research in Computer and Communication Engineering, Vol. 2, No. 10, April 20-21, 2015, pp. 2-5.

[20] Md. Shamsul Hoque, Md. Asif Imran, "Modification of Webster delay formula under non-lane based heterogeneous road traffic condition", Journal of Civil Engineering (IEB)", Issue 7, February 2007, pp. 1-4.

[21] Chu Cong MINH, Tran Hoai BINH, Tran Thanh MAI and Kazushi SANO, "The Delay Estimation Under Heterogeneous Traffic Conditions", Journal of the Eastern Asia Society for Transportation Studies, Vol. 7, February 2009, pp. 2-9.

[22] Md. Hadiuzzaman, Md. Mizanur, Tanweer Hasan and Md. A. Karim, "Development of Delay Model for Non-Lane Based Traffic at Signalized Intersection", Journal of Civil Engineering (IJCE), Vol. 3, Issue. 2, March 2014, pp. 69-79.

[23] Saoreen Rahman, Nilufar Yeasmin, Mahtab U. Ahmmed, M. S. Kaiser, "Adaptive Route Selection Support System Based On Road Traffic Information", 2nd International Conference on Electrical Engineering and Information and Communication Technology(ICEEICT), Bangladesh, May 21-23, 2015, pp. 2-4.

[24] V. K. Arasan, "Modeling Platoon Dispersal Pattern of Heterogeneous Road Traffic.Transportation Research Record", TRB, National Research Council, Washington DC, USA,S.H. (2003).

[25] R. Underwood, "Speed, volume, and density relationships: Quality and theory of traffic network", Yale bureau of highway traffic, Issue 6-7 (July 2003).

[26] G. Pang, K. Takahashi, T. Yokota, H. Takenaga, "Adaptive Route Selection for Dynamic Route Guidance System Based on Fuzzy-Neural Approaches ", 1995(IEEE), Japan.

[27] Sagheer Abbas, M. Saleem Khan, Khalil Ahmed, M.Abdullah and Umer Farooq, "Bio-inspired Neuro-Fuzzy Based Dynamic Route Selection to Avoid Traffic Congestion", International Journal of Scientific and Engineering Research, Vol. 2, Issue. 6, June 2011, pp. 2-4, ISBN:22295518.

[28] Yanfang Deng, Hengqing Tong, "Dynamic Shortest Path Algorithm in Stochastic Traffic Networks Using PSO Based on Fluid Neural Network", Journal of Intelligent Learning Systems and Applications, Vol. 3, February 2011, pp. 11-16.

[29] Dao Jiang, "A Novel Hybrid Optimization Algorithm based on Improved ACO and FNN", International Journal of Hybrid Information Technology, Vol. 9, April(2016) pp. 173-182, ISBN: 1738-9968.

[30] A.Renugambal , V.Adilakshmi Kameswari, "Finding Optimal Vehicular Route Based On GPS", International Journal of Computer Science and Information Technologies, Vol. 5(2), June (2016) pp. 1-3, ISBN: 13301332.

[31] Lili Cao , John Krumm, "From GPS Traces to a Routable Road Map", ACM GIS '09, Seattle, WA, USA, November 4-6, 2009 pp. 1-3, ISBN: 978-1-60558-649.

[32] Jie Liu, Bodhi Priyantha, Ted Hart, Heitor S. Ramos, Antonio A.F. Loureiro, Qiang Wang, "Energy Efficient GPS Sensing with Cloud Offloading", SenSys12, Toronto, ON, Canada., November 6-9, 2012 pp. 1-3, ISBN: 978-1-4503-1169-4.

[33] ME. Hossain, TN Turna, SJ Soheli, MS Kaiser, "Neuro-fuzzy(NF)based adaptive flood warning system for Bangladesh ", International Conference on Informatics, Electronics \& Vision (ICIEV), 2014, Dhaka, Bangladesh, 23-24, May 2014, pp. 1-3, ISBN:978-1-4799-5179-6.

[34] MS Kaiser, MH Chaudary, RA Shah, KM Ahmed, "Neuro-fuzzy (NF) based relay selection and resource allocation for cooperative networks", International Conference on Electrical Engineering/Electronics Computer Telecomunication and Information Technology (ECTI-CON), 2010, Chaing Mai, 19-21, May 2010, pp. 224-248, ISBN:978-1-42445607-9.

[35] MS Kaiser, ZI Chowdhury, S Al Mamun, A Hussain, M Mahmud, "A Neuro-Fuzzy Control System Based on Feature Extraction of Surface Electromyogram Signal for Solar-Powered Wheelchair", IEEE
Sympo. on Computational Intelligence in Healthcare and e-health (CICARE2015), Cape Town, South Africa, 16 August 2016, pp. 1-9.

[36] G.K.H. Pang, K. Takabashi, T. Yokota, "Prediction of Road Traffic Accidents in Jordan using Artificial Neural Network (ANN) ", Journal of Traffic and Logistics Engineering, Vol. 2, No. 2, Issue. June 2014, pp. 1-3.

[37] Khair S. Jadaan, Muaath Al-Fayyad, Hala F. Gammoh, "Adaptive route selection for dynamic route guidance system based on fuzzy-neural approaches", IEEE Transactions on Vehicular Technology, Vol. 48, Issue. 06 August 2002, pp. 1-3, Online ISSN: 1939-9359.

[38] Kranti Kumar, M. Parida, V.K. Katiyar, "Short term traffic flow prediction for a non urban highway using artificial neural network", $2^{\text {nd }}$ Conference of Transportation Research Group of India (2nd CTRG) ,Kranti Kumar et al. / Procedia - Social and Behavioral Sciences 104(2013)755764, 2013, pp. 757-759.

[39] R. Yasdi, "Prediction of Road Traffic using a Neural Network Approach", R. Neural Computing \& Applications, Springer-Verlag, 03 March 2014, pp. 1-3, ISSN:0941-0643.

[40] Fatma Al-Widyan, Nathan Kirchner, Michelle Zeibots, "An empirically verified Passenger Route Selection Model based on the principle of least effort for monitoring and predicting passenger walking paths through congested rail station environments", Australasian Transport Research Forum 2015 Proceedings, Sydney, Australia, 30 September - 2 October 2015, pp. 1-4. 